\title{
Organoids as Reliable Breast Cancer Study Models: An Update
}

\author{
Andrew Octavian Sasmita and Ying Pei Wong ${ }^{*}$ \\ Division of Applied Biomedical Sciences and Biotechnology, School of Health Sciences, International Medical \\ University, Malaysia
}

*Corresponding author: Ying Pei Wong, Division of Applied Biomedical Sciences and Biotechnology, School of Health Sciences, International Medical University, 57000 Kuala Lumpur, Malaysia

\begin{abstract}
Having been identified for centuries, breast cancer still remains one of the leading causes of death in women. Within the United States of America (USA) alone, it is estimated that over 60,000 women would have developed new cases of malignant breast cancer in 2017 alone; thus, calling an attention for the development of a reliable and effective breast cancer model for drug discovery to combat the disease or diagnostics to predict the progression of the disease. Current breast cancer models involve usage of 2D cell cultures and in vivo models which do not recapitulate the entire microenvironment of tumour growth in human cases, and despite some cell lines being derived from patients' own tumour cells, 2D cell cultures are heterogeneous and lack the cell-cell interaction necessary for normal tumour growth in the human body. Many limitations of these conventional models can be resolved using novel 3D organoid cultures produced from tumours of patients themselves or other viable sources. Breast cancer organoids have been proven to predict patient chemotherapeutic outcomes efficiently in the case of primary breast cancer via immunofluorescence or gene expression studies. This study model can also be utilized to discover new biomarkers with clinical implications. The advantages, limitations, and protocols to derive breast cancer organoids are being reviewed here alongside the ethical issues which might arise. Breast cancer organoid research could then open avenues of reliable drug discovery and diagnostics model to ameliorate subtypes of breast cancer which do not provide much options therapy-wise, such as the triple negative subtype.
\end{abstract}

\section{Keywords}

3D cell culture, Breast cancer, Organoid, Patient-derived xenografts, Spheroid

\section{Introduction}

The study of breast cancer has progressed massively over the past 2 decades, which raised public awareness of this disease as well as the quality of treatment. Despite the massive amount of effort, the effectiveness of clinically breast cancer chemotherapeutic drugs, including tamoxifen and anastrozole [1,2], as well as trastuzumab [3], a widely known drug which specifically targets human epidermal growth factor 2 (HER2) breast cancer, still varies from one patient to another $[3,4]$. Having understood how similarly but also differently the molecular progression of different patients' cases calls for the attention to develop a functional tailored biological model, with the goal of effectively curing this disease, one patient at a time.

Throughout the years, various breast cancer cell lines have been established including the widely used Michigan Cancer Foundation-7 (MCF-7) [5] and Monroe Dunaway Anderson (MDA-MB-231) [6,7] invasive ductal carcinoma cell lines amongst others. Despite the major breakthroughs these cancer cell lines in the drug discovery or understanding the basic molecular mechanism of disease progression, it has become apparent that 2D cultures of breast cancer is not enough to fully conclude the efficacies of different treatments in patients, especially in a dynamic disease which incorporate intercalating components $[8,9]$. Moreover, the use of animal models (e.g. rodents, cats, and dogs) [10] in the study of human breast cancer, albeit some physiological matches, still does not portray the disease as a whole [11,12]; thus, as medical sciences progress to more personalized variants of treatments, diagnostic and predictive models of breast cancer should also provide a more specific and holistic approach.

Turning away from conventional 2D cell lines, researchers started cultivation of $3 \mathrm{D}$ cell cultures, com- 
monly known as spheroids, popularized by the various works of Bissell, et al. which underlined the importance of tumour microenvironment which can be recapitulated by a more robust 3D cell culture [13]. Producible within hydrogel scaffolds $[14,15]$ or bioreactors $[16,17]$, these spheroids can be further developed to form the more in vivo-like organoids which express most components displayed by cancer cells, including malignant epithelial and endothelial cells aside from extracellular matrix (ECM) components $[12,18]$. This article will then review the rapidly emerging organoid models in breast cancer research alongside its advantages and limitations in the face of currently available 2D or animal models.

\section{Overview of Breast Cancer Molecular Subtypes}

Breast cancer is one of the first cancers to be researched vastly and the heterogeneous nature of this disease has been observed since before the dawn of molecular studies. Early histopathological findings have provided researchers with different physiological insights, including distinctions between ductal and lobular traits or the infiltration of metastases into the lymph nodes $[19,20]$. Currently, there are several molecular subtypes of breast cancer, all of which pose different clinical implications and outcome based on the course of treatment the patient undergoes. Based on the breakthrough provided by Sørlie, et al. with their microarray studies of ample complementary deoxyribonucleic acid (cDNA) from breast cancer samples, 5 molecular subtypes of breast cancer could be deduced [21]. These molecular subtypes differ in the grades of the tumour and presence (i.e. positive or negative) of oestrogen receptor (ER), progesterone receptor (PR), HER2, and Ki67 protein [21,22]. The status within each subtype is summarized in Figure 1 constructed below. Other studies yielded similar molecular classifications derived their grouping system via microarray analysis, such classifications were made in studies conducted by Munirah, et al. which discovered that majority of Malay ethnic breast cancer cases belong to the luminal A subtype, and unlike other subtypes in this classification, it is accompanied by negativity of the $\mathrm{p} 53$ protein [23]. Hu, et al. also supported the use of the intrinsic molecular classification by distinguishing 306 genes which could not only predict the distinct molecular subtypes, but also form strong conclusions on each subtype's overall survival rate [22].

The 5 molecular subtypes groupings are also characterized by the range of breast cancer 1 (BRCA1) and other oncogenic mutations which may be harboured differently in each subtype [24]. Aside from that, the molecular subtypes shown in Figure 1 can also be utilized as a prognostic tool, whereby breast cancer of the luminal subtypes which account for more than $50 \%$ of total cases are lower grade and present better prognosis as compared to the triple-negative subtype. Given the absence of 3 major receptors and a high correlation with breast cancer 1 (BRCA1) mutations, the options of

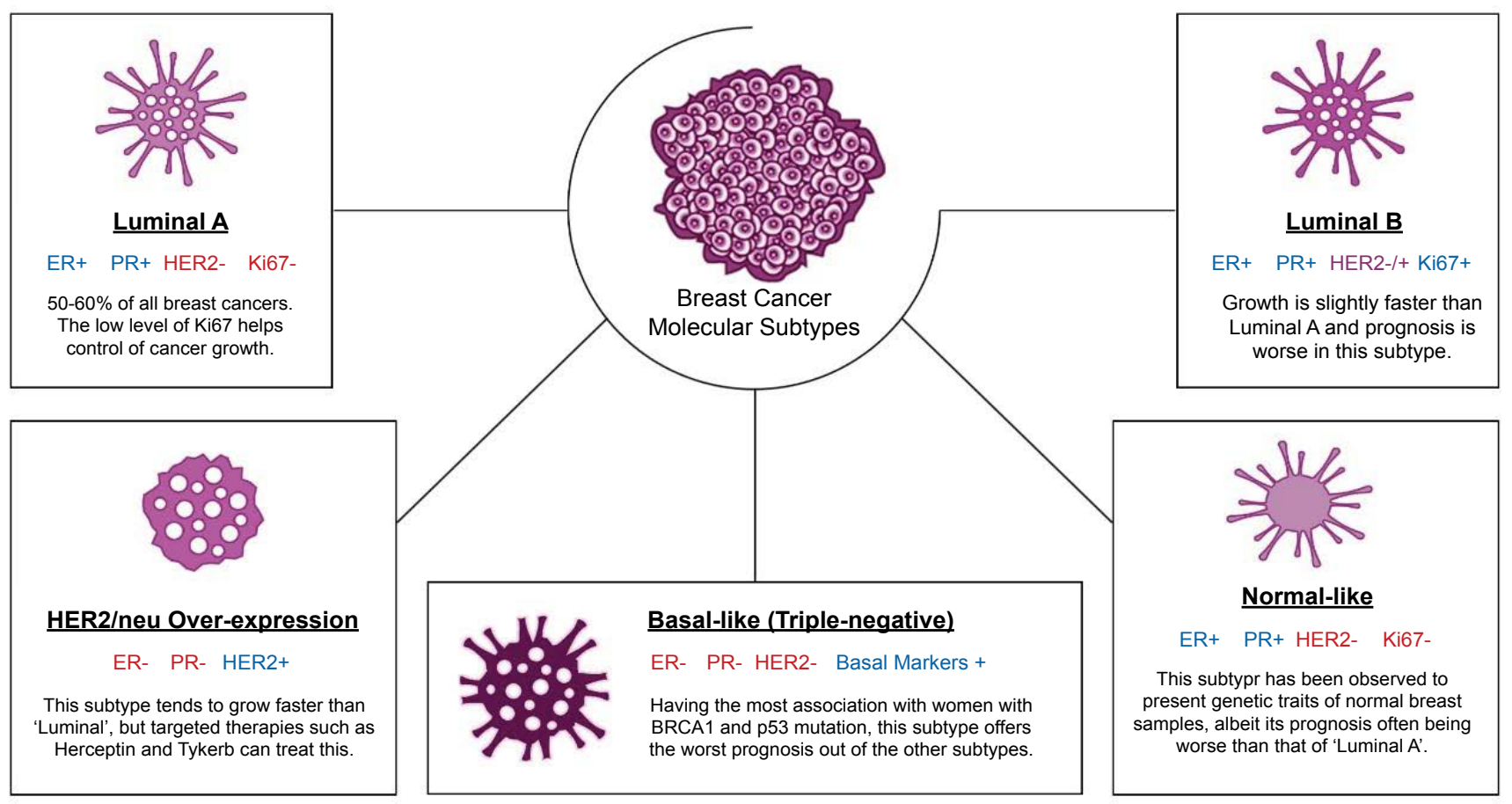

Prognosis Scale

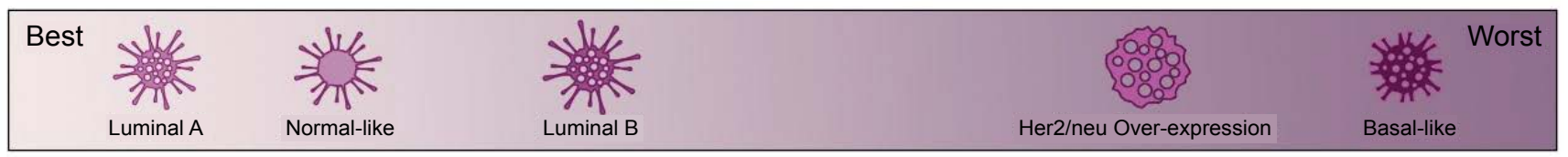

Figure 1: Molecular subtypes of breast cancer and their relative prognostic outcome. 
treatment for such patients are limited to a combinatorial radiation and chemotherapy or targeted therapies for non-HER2/neu patients [24].

There are however, differing opinions of some research groups around the world have allowed more classifications to surface, include the basal and non-basal subtypes of breast cancer or the molecular pathway classification $[25,26]$. Despite there are novel outlooks to molecular groupings of breast cancer, however this could negatively impact the development of treatment as there is no standardized method to predict the different classes [27]. The molecular subtypes are not only distinguishable via extensive laboratory-based microarray tests [21-23], but also other sophisticated genetic models which are available to diagnose the different types of breast cancer, such as MammaPrint [28] and Oncotype DX [29]. The availability of commercial molecular subtyping allows the prediction of a patient's cancer to a certain treatment and this prognostic power, if replicated correctly and efficiently to patient's own tumour-derived organoids will most likely increase the treatment success rate.

\section{Conventional Biological Models in Breast Cancer Research}

Having been studied extensively, various breast cancer cell lines and animal models have emerged throughout the years, each with its own distinctive traits. From the widely used MCF-7 cells to in vivo dog models, each model comes with series of advantages and limitations which are discussed in the following sub-sections. Despite the common usages of these models, it has become apparent that generic cancer models do not work optimally to predict each and every patient's conditions, let alone outcome [30].

\section{D cancer cell lines}

The emergence of 2D breast cancer cell lines could be traced back to 1958 whereby the first cultivated breast carcinomas named BT-20 was developed [9,31]. The pioneering culture study allowed various studies globally to come up with their own cell lines. To date, there are over 50 different cell lines have been established in the field of breast cancer research, some of which are widely and more commonly used in the research setting [32]. It is to be considered that these cancer cell lines may represent more than 1 particular molecular subtype or to a deeper extent, epigenetic profiles [33], while others were designed to recapitulate the hallmarks of particular subtypes.

MCF-7 cells have been widely utilized for the purpose of breast cancer drug discovery as well as the pathophysiological studies of breast cancer. Various studies have demonstrated that MCF-7 cells do in fact, belong to the luminal A subtype [33,34]. Despite majority of the claims that MCF-7 belongs to just 1 subtype, there have been questions rising on the validity of the molecular grouping, as it seems that other molecular subtypes exist within cultures of MCF-7 cells, namely triple-negative [35]. The triple-negative subtype of MCF-7 seemed to develop upon culturing of MCF-7 cells with fulvestrant [35], a type of hormone receptor positive treatment of breast cancer, which raises more questions on the possible morphological and molecular changes within stable breast cancer cell lines in the face of certain treatment options. One study conducted by Jiang, et al. [36] have found that protein expression levels of patient-derived tumour samples were more reproducible not in MCF-7 cells, but in the less commonly used T47D and BT483, again underlining the idea that one cell line does not fit all.

As opposed to the generic breast cancer cell lines, the use of primary cell cultures derived from patient's own tumour have been studied to give better prognostic power [37,38], however, discrepancies could be observed within cell lines that belong to the luminal subtype as they are being passaged. This phenotypic drift often favours the competitive growth of the more aggressive basal or triple-negative subtypes, would skew the results of drug testing on such cells, thus the use of at most, first-passage cells is recommended [36,37]. The spontaneous rise of triple-negative cells in this scenario could present problems of its own as there have been very few successful treatment options for triple-negative breast cancer patients despite the availability of generic cell lines which faithfully present the molecular markers of triple-negative breast cancer cells [39], which might give false negatives in studies which focus on another breast cancer subtype other than the triple-negative.

The use of 2D cell lines, be it derived from patient's own tumour or the use of commercially established cell lines, presents a good outlook from an economic standpoint as well as in reducing excessive usage of animal models [40]. Given that the transcriptomic and proteomic profiles do match the patients' tumours behaviour, the utilization of well-characterized cell lines could allow a faster and higher throughput screening of drugs [40]. The main limitations, however, include the several key factors within cancer biology that these 2D cell cultures do not portray or adhere to, including the stromal and matrix component of cancer out of the various other microenvironment factors [41,42]. More specifically in the case of generic cell lines, some studies have pointed out that established cancer cell lines display more genetic mutations and aberrations to those found in patient-derived tumours $[30,43,44]$. Cancer cell lines are also prone to cross-contamination and the use of such cell lines could lead to catastrophic outcomes if not validated [45].

\section{In vivo models}

The use of animal models within biological research is as common as the use of cell lines. The main argu- 
ment for a cancer study to utilize animal models would be that whole organisms could display the complexity of intricate diseases like cancer which gives the research a better understanding compared to the use of in vitro $2 \mathrm{D}$ cell cultures.

Having been utilized for various other diseases, mice models of breast cancer have been utilized copiously within the field. The clear evidence that a more robust and cost-effective diagnostic model is needed can be further emphasized by the slow emergence of functional therapies or the translational effects in patients' cases, with only lesser than $10 \%$ of drugs being screened daily making it past early phases of development $[11,46]$. To test the drug trastuzumab's response in alone, a meta-analysis conducted by Chen, et al. in 2016 has revealed at least 80 mice model studies using over 2000 mice in the sets of experiments [47]. Out of the thousands of preclinical mice models, many different variations exist, each bearing their own sets of benefits and drawbacks.

In syngeneic models, breast cancer cell lines are allografted within immunocompetent hosts. The main importance of syngeneic models in cancer research is the intact immunity that is present, unlike in immune-deficient models, and this pose huge implications especially in targeted therapies which are immunological-based [48]. Despite the absence of tumour resection from syngeneic such as one conducted by Bailey-Downs, et al. in BALB/cj mice [49], it has been noted that other studies have reported a significant immunogenic reaction which leads to regression of tumour growth [48]. Other important limitations are the short timespan for treatment in syngeneic models as some tumours exhibit $100 \%$ development of the primary tumour, albeit being very aggressive $[49,50]$, and the murine allografts which are different than human-derived tumour cells and thus, species differences might occur [51].

As a combinatorial effort to attain better portrayal of the disease, breast cancer cells might also be embedded as xenografts within mice models. The cells being xenografted into the immune-deficient models can be of immortalized human breast cancer cell lines or patient-derived xenografts (PDX), and these allow the recapitulation of tumour microenvironment at a more specific level, given that it is grown within a whole organism $[48,50]$. In the use of immortalized cell lines however, there has been a notable lack of heterogeneity that can be observed in the case of patient-derived tumour xenografts [51]. Some of the widely studied xenografts are the HER2/neu mouse models, such as PDX in non-obese diabetic severe compromised immunodeficiency (NOD/ SCID) mice in a study conducted by Liu, et al. [52] and $\mathrm{NOD} / \mathrm{SCID}$ gamma (NSG) mice conducted by lorns, et al. [53], both of which studied the metastatic type of breast cancer.

Lastly, in vivo models of breast cancer may under- go genetic modifications, producing transgenic models termed as genetically modified mice models (GEMM) which allow the induction of tumour development within the original tissue of the organism itself. Much like the syngeneic models, the biological models' immune system will remain and the GEMMs can be induced by the mouse mammary tumour virus (MMTV) [54] or using doxycycline-inducible promoters [55]. The most limiting factor in developing GEMMs for breast cancer is that it is time-consuming and costly $[48,56]$. Recent molecular developments however, such as RNA interference (RNAi) via short hairpin ribonucleic acid (shRNA) [57] and clustered regularly interspaced short palindromic repeats caspase 9 (CRISPR/Cas9) systems $[58,59]$ allow a more rapid induction of such genetic changes, some of which are even applied in constructing robust organoid models discussed in later sections.

Generally, animal models require more capital to maintain and takes longer to give the desired research output as compared to 2D cell cultures, albeit being more thorough and robust. Factors such as difficulties in obtaining quantitative data and animal stress also comes into play with the use of animal models [41], with the latter also correlating to human stress to a worse prognosis in metastatic cancers. Cancer occurs at varying heterogeneity histologically and genomically between mouse models and human tumours [60], and thus the use of a more practical in vitro model is preferable and required for a higher throughput screening of drugs aside from various other applications.

\section{3-D Organoid Models}

Organoid cultures have been used in cancer research to model gastrointestinal [61], lung [62], or even prostate cancers [63]. This section will review the recent advances in developing breast cancer organoids alongside their uses and other limitations. Table 1 at the end of this section summarizes relevant studies and findings in breast cancer organoid research while Table 2 summarizes the advantages, limitations, and possible solutions pertaining to breast cancer organoids.

\section{Development of breast organoids}

Deriving their methods from experimental studies and modified protocols of organoid generation of other organs, researchers are continuously optimizing protocols to develop breast cancer organoid cultures. In this article, various sources of breast cancer organoids with relevant protocols are reviewed.

Breast cancer organoids from primary breast tumour tissues: The first basic protocol for breast cancer organoid development involve the use of primary tumour samples have been developed by DeRose, et al. in 2013 [64]. The tumour samples were dissociated mechanically and chemically. The incubation time for digestion varies, whereby human-derived tumours require at least overnight incubation whilst mice graft-derived 
Table 1: List of current researches being done on breast cancer organoids as study models.

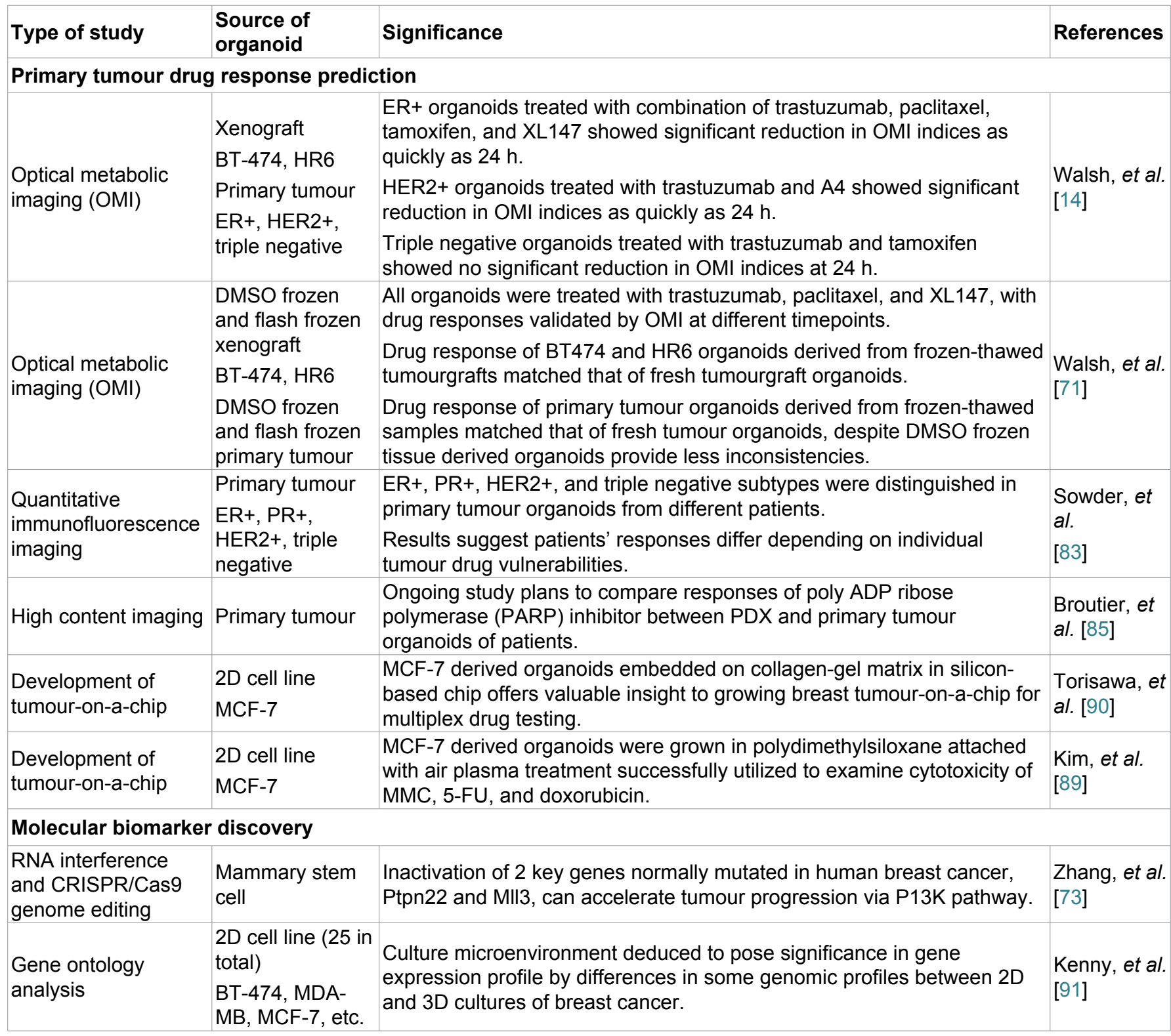

Table 2: Advantages, limitations, and reported solutions of organoids as study models.

\begin{tabular}{|c|c|c|}
\hline Advantages & Limitations & Reported solutions \\
\hline $\begin{array}{l}\text { Outlet to study human diseases which are } \\
\text { difficult to study in animal models. }\end{array}$ & $\begin{array}{l}\text { Loss of oncogene drive in rich growth factor } \\
\text { conditions [37]. }\end{array}$ & $\begin{array}{l}\text { Opt to use Matrigel/hydrogel devoid of } \\
\text { growth factors [64]. }\end{array}$ \\
\hline $\begin{array}{l}\text { More complete profile of disease } \\
\text { compared to 2D cell lines [91]. }\end{array}$ & Cost and difficulty to scale-up [95]. & $\begin{array}{l}\text { Development of hanging drop } \\
\text { protocols or magnetic cell levitation. }\end{array}$ \\
\hline $\begin{array}{l}\text { Faster reaction endpoints and quantitative } \\
\text { data collection compared to animal studies } \\
{[14,41,64,71] \text {. }}\end{array}$ & $\begin{array}{l}\text { Weak vascularization leading to hypoxia } \\
\text { which triggers activation of genes to mediate } \\
\text { cell survival [94]. }\end{array}$ & $\begin{array}{l}\text { Hypoxic conditions have been } \\
\text { displayed in vivo and thus, might be } \\
\text { its natural phenotype [95]. }\end{array}$ \\
\hline \multicolumn{3}{|l|}{$\begin{array}{l}\text { More practical for immunofluorescence } \\
{[14,71] \text {. }}\end{array}$} \\
\hline $\begin{array}{l}\text { Derivable from various tissue sources. } \\
\text { Fibrinogen and fibroblasts which can } \\
\text { cross-link with PEG to form hydrogel can } \\
\text { be used to study metastasis based on } \\
\text { stiffness of product }[92,93] \text {. }\end{array}$ & $\begin{array}{l}\text { Murine origin of Matrigel might not be } \\
\text { compatible to be transplanted back into } \\
\text { humans [97]. }\end{array}$ & $\begin{array}{l}\text { Use other types of matrix which are } \\
\text { compatible to humans (e.g. hydrogel). }\end{array}$ \\
\hline Phenotype retained for up to 70 days [74]. & $\begin{array}{l}\text { Rigidity of ECM component might dampen } \\
\text { drug molecules introduced [93]. }\end{array}$ & \\
\hline
\end{tabular}

tumours require shorter time [64,65]. Digestions carried out with lower enzymatic concentrations at longer timepoints have also been observed to yield more viable organotypic cultures [66].
Once organotypic cultures have been initiated, it may take as fast as $24 \mathrm{~h}$ for the culture to reach $75 \%$ confluency, which is the amount recommended by Garbe, et al. to seed the cells optimally [65]. Organoids may 
not attach in the first seeding, thus adding Y27632 dihydrochloride or fasudil, which are stem cell survival promoters, may protect organoids from anoikis before seeding on new culture vessel if required $[66,67]$.

These organotypic cultures might be subjected to lentivirus introduction in metastatic studies of breast cancer as transduction has been reported to be low in primary organoid cultures $[64,68]$. Once organoid cultures have matured or transduced, single cells are further separated from the culture by differential centrifugation followed by careful aspiration of the supernatant. The pellet is then resuspended in medium which can accommodate growth of mammary epithelial cells, such as M87 medium [64]. Matrigel at $4{ }^{\circ} \mathrm{C}$ was then added into the organoid suspension in the culture vessel and the vessel would then be inverted to let the Matrigel to solidify and not entrap the organoids at the bottom of the 3D structure. There is no measure of timepoint for organoid growth aside from reaching their desired confluency of more than $70 \%$. There are however, factors that may affect speed of growth of organoids, including cell concentration and nutrition available within medium [64]. Incorporation of growth factors, which is absent in DeRose's study, may promote faster growth of organoid cultures [66].

Once established, the solidified organoid within Matrigel or other matrix could be visualized via immunofluorescence studies to observe viability, presence of breast cancer markers on the surface of organoids, including cytokeratin-14 (K14), cytokeratin-19 (K19), or p63 [66,69] with the nuclei counterstained with Hoechst or Rhodamine $[66,70]$, which can indicate the degree of invasiveness of breast cancer, as well as presence of hormone receptors via metabolic imaging studies [71]. Walsh, et al. has successfully shown the growth of primary tumour samples obtained from xenografts and not just human samples in 2014 [71] and 2016 [14]. To a lesser extent, 2D cell lines could also be utilized as the core of these 3D organoid structures as opposed to patient-derived tissues [72], but the implication of this type of study could only be used for the study of the different molecular subtypes of breast cancer as they do not portray patient's own subtypes.

These established 3D organoids could also be utilized for in vitro drug tests, with applications discussed in later sections. Distinguishable from cell lines, primary tumour cells from human samples also grow significantly slower and thus proliferative studies might be carried out at longer periods. For maintenance and storage, organoids can be cryopreserved prior to adding medium and embedding it into the Matrigel matrix, or if organoids need to be maintained in $37^{\circ} \mathrm{C}$ incubators for upcoming studies, medium needs to be replaced at least 3 times within 1 week $[64,66]$.

Breast cancer organoids from mammary stem cells: With the CRISPR/Cas9 genome editing technique,
Zhang, et al. have shown that molecular inactivation of 2 breast cancer related genes, namely Ptpn22 and Mll3 can promote tumorigenesis from mammary stem cells (MaSC), whereby the phenotype has been carried out in the formation of 3D organoid cultures [73]. These organoids were transduced via lentiviral vectors containing shRNA which was then implanted into GEMMs, promoting growth of tumours resembling HER2 overexpression molecular subtype. Another study which alters activation of Wnt and HER2/neu signaling within MaSC conducted by Jarde, et al. has shown to give promising results in growing 3D organoids within Matrigel matrix which resemble a less used subtype of breast cancer, Wnt1 [74,75].

Possessing various genes that may be altered, the coupling of genomic editing in MaSC has proven to be a viable option to generate tumours which can be utilized to grow 3D organoid structures. These organoids may even be grown for 30 or more days to develop the distinguishing factors of mammary gland and breast tissues [74], including the nodules as well as ductal and luminal vessels, which would give a better outlook on the mechanism of drugs being tested. Wu, et al. studied MaSC obtained from marmosets [76] and found that breast cancer organoids formed from these MaSC resemble the phenotype of human cancers, whereby they are solid and spherical for both luminal and basal subtypes, unlike in organoids derived from mice tumour grafts, which may yield hollow organoids for luminal subtype [77].

Breast organoids from normal breast tissues: With similar steps to initiate organotypic cultures from primary breast tumour tissues in the previous sub-section explained by DeRose, et al. [64], the generation of organoids from normal breast tissues require normal breast samples of patients, which have been explained by Sokol, et al. in 2016 [78]. The derivation of organoids from this step require the administration of lentiviral vectors to transduce the cells to multiply much like in the development of intestinal organoids [79] and subsequent growth in hydrogel matrix alongside growth factor load. Although not applicable to study the progression of cancer in personalised cases per se, normal breast organoids have been utilized to study the extravasation of blood vessels as well as studies to compare biomarkers present in tumours and normal breast tissues [80].

\section{Organoid use as breast cancer model}

Breast cancer organoids have been reported to possess ranging applications. The various applications from recent researches utilizing breast cancer organoids are reviewed in this sub-section.

Primary tumour drug response prediction: The most desirable application of breast cancer organoids is their ability to predict the course and efficacy of treatment at a personalized scale. In the optical metabolic imag- 
ing (OMI) of primary tumour organoids which are ER+ and HER2- after comparison with the OMI profile of BT474 and HR6 organoids, results shown by the ranging fluorescence intensity indicate that treatment with trastuzumab does not change the OMI index, as well as varying response upon treatment of paclitaxel and tamoxifen, which can be clinically validated [71,81,82]. Moreover, combinatorial treatment of paclitaxel, trastuzumab, and XL14 to result in a significant reduction of OMI index for both cell line-derived organoids and primary tumour organoids from patient's own fresh sample.

Another research discussed by Sowder, et al. has shown yet another in vitro organoid culture which could be observed to express all the molecular subtypes of breast cancer heterogeneously and thus display different vulnerabilities towards current treatment based on the subtypes [83]. This in turn gives a more robust understanding on how organoids can be utilized to recapitulate not only the tumour microenvironment but also molecular subtypes of patient's tumours and thus increasing the prognostic value of this model. Within the various studies, it has been noted that the varying results, albeit being a few, may indicate the presence of cancer stem cells (CSC)-like cells which might confer to the primary tumour's drug resistance as well as renewal abilities [84].

A completed study carried out by Broutier, et al. focused on translating PDX organoids into the field of prognostics and diagnostics [85], a project which could lead to ease the pipeline within breast cancer drug discovery and to minimize the cost and time taken for these treatments to reach patients. Once established, this could be an upgraded form of the currently existing breast cancer explant biobank studied by Bruna, et al. [86], which harbours hundreds of PDX models, capable of undergoing high-throughput drug screens as well as comparative studies with the normal breast tissue samples of the same patient. This model, despite not being organoid-based, is very much like a colorectal cancer biobank constructed by van de Wetering, et al. [87], which could be the next step by incorporating organoid models in place of breast cancer PDX models alone in the biobank.

Lastly, for multiplex drug testing, researchers have come up with tumours on a chip, which is yet another innovative take on diagnostics and drug screening methodologies, whereby Torisawa, et al. [88] and Kim, et al. [89] showed protocols to create 3D cultures of breast cancer cells suspended on microchips with microfluidic channels. Cytotoxicity results of different anticancer treatments such as mitomycin C (MMC) and 5-fluorouracil (5-FU) have been reportedly comparable when grown on this chip and in MTT assay [90], although it was also observed that the growth rate of the 3D cell culture was markedly slower than 2D cell culture.
Molecular biomarker discovery: The discovery of key molecular determinants of breast cancer is important, especially in the face of genomic editing techniques, CRISPR/Cas9 or shRNA in correcting these aberrations and possibly offering us with a treatment outlet. Breast cancer organoids generated from MaSC transplanted in GEMM have provided a molecular outlook on inactivation of cancer-related genes via the CRISPR/ Cas9 method which appear to be mutated in human breast cancer cases in general, namely Ptpn22 and Mll3 [73]. Compared to 2D cell lines whereby high throughput drug screens are mostly performed at, some genetic aberrations are over-represented while not being so in 3D cultures.

Within a gene expression array study, it is emphasized that signal transducers and regulatory enzyme activities are statistically over-represented in 2D cell lines and not in 3D cell cultures when compared to in vivo model [91]. The research group study also found that despite the molecular subtypes playing a major role in gene expression profiles, there still is importance of how tumour microenvironment and culture conditions affecting genomic profiles significantly [91]. Biomarker discovery in the end, still proved to be a necessary field in breast cancer research as there has been reported various shifts at a molecular level to another subtype upon 1 type of treatment regimen [35], not to mention the scarce treatment option for aggressive triple-negative basal like cancers.

\section{Advantages of 3D breast cancer organoids}

We first consider the patient-derived organoids as an outlet to study human disease progressions which are otherwise difficult to study in animal models. As compared to 2D cell lines, organoids provide a more complete and thorough picture of the progression of the disease, including phenotypic similarities and biomarker profile when compared to patient's own tumours [92]. Three-dimensional cell cultures of breast cancer also show faster timepoint to gather quantitative data as compared to animal studies $[14,41,64,71]$, and the in vitro properties of organoids makes it a more practical choice in immunofluorescence studies [14,71].

Organoids offer a biological model which can be derived from various sources of tissues, including normal tissues or tumours from adult patients, as well as embryonic stem cells (ESC) and induced pluripotent stem cells (iPSC), whereby when propagated to its optimum potential, will portray the disease alongside its microenvironment component at a high degree. It has been portrayed that fibrinogen and fibroblast components produced by metastatic breast cancer can cross react with polyethylene glycol (PEG) to form hydrogel, thus, the solid and stiff nature that organoids can grow into may also indicate the degree of metastatic breast cancer in experimental models [92,93]. Organoids grown for extensive period are stable such as shown by Jarde, et al. 
retained their phenotypes for up to 70 days since establishment of the mammary organoid culture, although the cell architecture seems to be lost accompanied by accumulation of chromosomal aberrations after 112 days upon establishment [74].

\section{Limitations of 3D breast cancer organoids}

One of the major concerns of growing organoids as a study model in cancer research is that the loss of oncogene drive due to the growth factor-rich environment which are dubbed enough to sustain the fastened proliferation [37]. In the case of the protocol that was discussed vastly by DeRose, [64], however, the Matrigel is devoid of these growth factors and thus, such limitations should not hinder the oncogenic drive. Despite mimicking most cellular architecture and surface markers faithfully, 3D organoids have been reported to have weak vascular organization which leads to hypoxia and subsequent activation of genes which modulate drug sensitivity alongside cell survival [94]; however, several studies have indicated that this drug resistance mechanism occurs within in vivo models as well, providing organoids with natural tumour behaviour to avoid over-representation of drugs upon screens [95]. Other limitations include cost and difficulty to scale-up aside from the rigidity of the ECM component and types of scaffold used $[95,96]$, which might indicate invasiveness or dampen incoming drug molecules, thus affecting drug tests. The future of implantation of genetically corrected organoids into humans as a therapeutic agent may also be affected by Matrigel that is widely used in the organoid scaffold structure, as it is of murine origin [97], and thus use of other types (e.g. hydrogel) might be preferable in the future use in human transplantation.

\section{Ethical issues}

Much like live tissue or embryonic stem cell samples, patient-derived organoids such as ones deposited in live biobanks [87] come with a series of possible ethical issues. The first ethical issue is the consent requested from the patients, whether it would be a broad, tiered, or specific consent. With the novel nature of organoid research, patients might feel justified with a broad consent [98], whereby they can withdraw their participation completely, or with tiered consent whereby they can choose what their tissues can be used for from a range of research outcomes [99]. Privacy is another issue whereby the institute receiving the tissue samples should always implement strategies to keep patient information confidential. Furthermore, property rights shall be considered, as given that the tissue comes from patients, but it will subsequently undergo modifications that it might not be considered as patient's own tissue anymore, a question asks about the proprietor of the submitted tissue itself [100]. Ethics play a huge role in any case of tissue submission; thus, research institutes or bodies should come up with strategies to mediate the ethics that come into play so that the advancements in organoid studies will not be hindered.

\section{Future Directions}

The development of a breast cancer organoid biobank is indeed an interesting prospect, allowing patients who require diagnostic or prognostic procedures done to do so with more ease. Given the heterogeneous nature of breast cancer itself, researchers have yet to come up with a generalized protocol to generate patient's primary tumour organoids and thus more studies should be conducted. The future of organoids, if coupled with genetic modification techniques such as the CRISPR/Cas9, can offer viable therapeutic options in different instances. Study has shown that epithelial organoids generated from gastrointestinal stem cells could be functionally repaired by genetic modification to revert the effects of a single locus mutation within the cystic fibrosis transmembrane conductor receptor (CFTR) [101]. This opens possibilities of the clinical applications of genetically corrected organoid cultures to be implanted in patient tissues to correct diseases, one of which could be breast cancer.

\section{Conclusion}

It has become apparent that despite the glories that $2 \mathrm{D}$ and in vivo models have provided us, there is a dire need for a more robust translational model to study breast cancer. With current researches abiding by different protocols to develop various types of breast cancer organoids, this model offers versatile approaches to develop it, but there still needs to be a general protocol to optimize production and yield of organoid culture as a guideline. The current applications of 3D cultures of breast tumours range from multiplex drug screening to predicting the course of treatment in a personalized case of a patient, and with the dawn of genomic editing techniques and its similar applications in colorectal cancer or gastrointestinal cancer organoids, researchers might even couple these techniques to form a pioneering therapeutic option for breast cancer.

\section{References}

1. Brauch H, Schwab M (2014) Prediction of tamoxifen outcome by genetic variation of CYP2D6 in post-menopausal women with early breast cancer. Br J Clin Pharmacol 77: 695-703.

2. Ingle JN, Buzdar AU, Schaid DJ, Goetz MP, Batzler A, et al. (2010) Variation in anastrozole metabolism and pharmacodynamics in women with early breast cancer. Cancer Res 70: 3278-3286.

3. von der Heyde S, Wagner S, Czerny A, Nietert M, Ludewig $F$, et al. (2015) mRNA profiling reveals determinants of trastuzumab efficiency in HER2-positive breast cancer. PLoS One 10: e0117818.

4. Januszewski A, Tanna N, Stebbing J (2014) Ethnic variation in breast cancer incidence and outcomes-the debate continues. Br J Cancer 110: 4-6.

5. Lee AV, Oesterreich S, Davidson NE (2015) MCF-7 Cells-- 
changing the course of breast cancer research and care for 45-years. J Natl Cancer Inst 107.

6. SL M, VM E, Brantley E (2016) Cytotoxicity in MCF-7 and MDA-MB-231 breast cancer cells, without harming MCF10A healthy cells. J Nanomed Nanotechnol 07.

7. Hsieh TC, Elangovan S, Wu JM (2010) Differential suppression of proliferation in MCF-7 and MDA-MB-231 breast cancer cells exposed to alpha-, gamma- and delta-tocotrienols is accompanied by altered expression of oxidative stress modulatory enzymes. Anticancer Res 30: 4169-4176.

8. Burdall SE, Hanby AM, Lansdown MRJ, Speirs V (2003) Breast cancer cell lines: Friend or foe? Breast Cancer Res 5: 89-95.

9. Holliday DL, Speirs V (2011) Choosing the right cell line for breast cancer research. Breast Cancer Res 13: 215

10. Cekanova M, Rathore K (2014) Animal models and therapeutic molecular targets of cancer: Utility and limitations. Drug Des Devel Ther 8: 1911-1921.

11. Clarke $R$ (2009) The role of preclinical animal models in breast cancer drug development. Breast Cancer Res 11: S22.

12. Katt ME, Placone AL, Wong AD, Xu ZS, Searson PC (2016) In vitro tumor models: Advantages, disadvantages, variables, and selecting the right platform. Front Bioeng Biotechnol 4 .

13. Gudjonsson T, Rønnov Jessen L, Villadsen R, Bissell MJ, Petersen OW (2003) To create the correct microenvironment: Three-dimensional heterotypic collagen assays for human breast epithelial morphogenesis and neoplasia. Methods 30: 247-255

14. Walsh AJ, Cook RS, Sanders ME, Arteaga CL, Skala MC (2016) Drug response in organoids generated from frozen primary tumor tissues. Sci Rep 6.

15. Song H HG, Park KM, Gerecht S (2014) Hydrogels to model 3D in vitro microenvironment of tumor vascularization. Adv Drug Deliv Rev 79-80: 19-29.

16. Massai D, Isu G, Madeddu D, Cerino $G$, Falco $A$, et al (2016) A versatile bioreactor for dynamic suspension cell culture. Application to the culture of cancer cell spheroids. PLoS One 11: e0154610.

17. Barrila J, Radtke AL, Crabbé A, Sarker SF, Herbst Kralovetz MM, et al. (2010) Organotypic 3D cell culture models: Using the rotating wall vessel to study host-pathogen interactions. Nat Rev Microbiol 8: 791-801.

18. Holle AW, Young JL, Spatz JP (2016) In vitro cancer cellECM interactions inform in vivo cancer treatment. Adv Drug Deliv Rev 97: 270-279.

19. Fisher CJ, Egan MK, Smith $P$, Wicks K, Millis RR, et al. (1997) Histopathology of breast cancer in relation to age. Br J Cancer 75: 593-596.

20. Schnitt SJ (2010) Classification and prognosis of invasive breast cancer: From morphology to molecular taxonomy. Mod Pathol 23: S60-S64.

21. Sørlie T, Perou CM, Tibshirani R, Aas T, Geisler S, et al (2001) Gene expression patterns of breast carcinomas distinguish tumor subclasses with clinical implications. Proc Natl Acad Sci U S A 98: 10869-10874.

22. Hu Z, Fan C, Oh DS, Marron J, He X, et al. (2006) The molecular portraits of breast tumors are conserved across microarray platforms. BMC Genomics 7: 96.

23. Munirah MA, Siti Aishah MA, Reena MZ, Sharifah NA, Ro- haizak M, et al. (2011) Identification of different subtypes of breast cancer using tissue microarray. Rom $\mathrm{J}$ Morphol Embryol 52: 669-677.

24. Dai X, Li T, Bai Z, Yang Y, Liu X, et al. (2015) Breast cancer intrinsic subtype classification, clinical use and future trends. Am J Cancer Res 5: 2929-2943.

25. Lehmann BD, Bauer JA, Chen X, Sanders ME, Chakravarthy $A B$, et al. (2011) Identification of human triple-negative breast cancer subtypes and preclinical models for selection of targeted therapies. J Clin Invest 121: 2750-2767.

26. Gatza ML, Lucas JE, Barry WT, Kim JW, Wang Q, et al. (2010) A pathway-based classification of human breast cancer. Proc Natl Acad Sci U S A 107: 6994-6999.

27. Pusztai L, Mazouni C, Anderson K, Wu Y, Symmans WF (2006) Molecular classification of breast cancer: Limitations and potential. Oncologist 11: 868-877.

28. Slodkowska EA, Ross JS (2009) MammaPrint 70-gene signature: Another milestone in personalized medical care for breast cancer patients. Expert Rev Mol Diagn 9: 417-422.

29. Roberts MC, Weinberger M, Dusetzina SB, Dinan MA Reeder Hayes KE, et al. (2016) Racial variation in the uptake of oncotype DX testing for early-stage breast cancer. $J$ Clin Oncol 34: 130-138.

30. Vargo Gogola T, Rosen JM (2007) Modelling breast cancer: One size does not fit all. Nat Rev Cancer 7: 659-672.

31. Lasfargues Ey, Ozzello L (1958) Cultivation of human breast carcinomas. J Natl Cancer Inst 21: 1131-1147.

32. Prat A, Karginova O, Parker JS, Fan C, He X, et al. (2013) Characterization of cell lines derived from breast cancers and normal mammary tissues for the study of the intrinsic molecular subtypes. Breast Cancer Res Treat 142: 237-255.

33. Kao J, Salari K, Bocanegra M, Choi YL, Girard L, et al. (2009) Molecular profiling of breast cancer cell lines defines relevant tumor models and provides a resource for cancer gene discovery. PLoS One 4: e6146.

34. Subik K, Lee JF, Baxter L, Strzepek T, Costello D, et al. (2010) The expression patterns of ER, PR, HER2, CK5/6, EGFR, Ki-67 and AR by immunohistochemical analysis in breast cancer cell lines. Breast Cancer (Auckl) 4: 35-41.

35. Leung E, Kim JE, Askarian Amiri M, Finlay GJ, Baguley BC (2014) Evidence for the existence of triple-negative variants in the MCF-7 breast cancer cell population. Biomed Res Int 1-7.

36. Jiang G, Zhang S, Yazdanparast A, Li M, Pawar AV, et al. (2016) Comprehensive comparison of molecular portraits between cell lines and tumors in breast cancer. BMC Genomics 17: 525

37. Hines WC, Yaswen P, Bissell MJ (2015) Modelling breast cancer requires identification and correction of a critical cell lineage-dependent transduction bias. Nat Commun 6: 6927.

38. Goodspeed A, Heiser LM, Gray JW, Costello JC (2016) Tumor-derived cell lines as molecular models of cancer pharmacogenomics. Mol Cancer Res 14: 3-13.

39. Grigoriadis A, Mackay A, Noel E, Wu P, Natrajan R, et al. (2012) Molecular characterisation of cell line models for triple-negative breast cancers. BMC Genomics 13: 619.

40. Hickman JA, Graeser R, de Hoogt R, Vidic S, Brito C, et al. (2014) Three dimensional models of cancer for pharmacology and cancer cell biology: Capturing tumor complexity in vitro/ex vivo. Biotechnol J 9: 1115-1128. 
41. Pampaloni F, Reynaud EG, Stelzer EHK (2007) The third dimension bridges the gap between cell culture and live tissue. Nat Rev Mol Cell Biol 8: 839-845.

42. Ferreira D, Adega F, Chaves R (2013) The importance of cancer cell lines as in vitro models in cancer methylome analysis and anticancer drugs testing. Oncogenomics Cancer Proteomics.

43. Domcke S, Sinha R, Levine DA, Sander C, Schultz N (2013) Evaluating cell lines as tumour models by comparison of genomic profiles. Nat Commun 4: 2126.

44. Huang Y, Liu Y, Zheng C, Shen C (2017) Investigation of cross-contamination and misidentification of 278 widely used tumor cell lines. PLoS One 12: e0170384.

45. Vantangoli MM, Madnick SJ, Huse SM, Weston P, Boekelheide K (2015) MCF-7 human breast cancer cells form differentiated microtissues in scaffold-free hydrogels. PLoS One 10: e0135426.

46. Kinders R, Parchment RE, Ji J, Kummar S, Murgo AJ, et al (2007) Phase 0 clinical trials in cancer drug development: From FDA guidance to clinical practice. Mol Interv 7: 325334.

47. Chen J, Yang C, Guo B, Sena ES, Macleod MR, et al (2016) The efficacy of trastuzumab in animal models of breast cancer: A systematic review and meta-analysis. PLoS One 11: e0158240.

48. Manning HC, Buck JR, Cook RS (2016) Mouse Models of breast cancer: Platforms for discovering precision imaging diagnostics and future cancer medicine. J Nucl Med 57: 60S-68S.

49. Bailey Downs LC, Thorpe JE, Disch BC, Bastian A, Hauser PJ, et al. (2014) Development and characterization of a preclinical model of breast cancer lung micrometastatic to macrometastatic progression. PLoS One 9: e98624.

50. Dranoff G (2012) Experimental mouse tumour models: What can be learnt about human cancer immunology? Nat Rev Immunol 12: 61-66.

51. Murphy J (2015) Pre-clinical murine models: Syngeneic models for immuno-oncology. MOJ Immunol 2.

52. Liu H, Patel MR, Prescher JA, Patsialou A, Qian D, et al. (2010) Cancer stem cells from human breast tumors are involved in spontaneous metastases in orthotopic mouse models. Proc Natl Acad Sci U S A 107: 18115-18120.

53. lorns E, Drews Elger K, Ward TM, Dean S, Clarke J, et al. (2012) A new mouse model for the study of human breast cancer metastasis. PLoS One 7: e47995.

54. Fry E, Taneja P, Inoue K (2016) Clinical applications of mouse models for breast cancer engaging HER2/neu. Integr Cancer Sci Ther 3: 593-603.

55. Fantozzi A, Christofori G (2006) Mouse models of breast cancer metastasis. Breast Cancer Res 8: 212.

56. Kersten $\mathrm{K}$, de Visser KE, van Miltenburg MH, Jonkers $\mathrm{J}$ (2017) Genetically engineered mouse models in oncology research and cancer medicine. EMBO Mol Med 9: 137-153.

57. Dow LE, O'Rourke KP, Simon J, Tschaharganeh DF, van Es JH, et al. (2015) Apc restoration promotes cellular differentiation and reestablishes crypt homeostasis in colorectal cancer. Cell 161: 1539-1552.

58. Huijbers IJ, Del Bravo J, Bin Ali R, Pritchard C, Braumuller TM, et al. (2015) Using the GEMM-ESC strategy to study gene function in mouse models. Nat Protoc 10: 1755-1785.

59. Annunziato S, Kas SM, Nethe M, Yücel H, Del Bravo J, et al. (2016) Modeling invasive lobular breast carcinoma by CRISPR/Cas9-mediated somatic genome editing of the mammary gland. Genes Dev 30: 1470-1480.

60. Hollern DP, Andrechek ER (2014) A genomic analysis of mouse models of breast cancer reveals molecular features of mouse models and relationships to human breast cancer. Breast Cancer Res 16: 59

61. Matano M, Date S, Shimokawa M, Takano A, Fujii M, et al. (2015) Modeling colorectal cancer using CRISPR-Cas9-mediated engineering of human intestinal organoids. Nat Med 21: $256-262$.

62. Nadkarni RR, Abed S, Draper JS (2016) Organoids as a model system for studying human lung development and disease. Biochem Biophys Res Commun 473: 675-682.

63. Drost J, Karthaus WR, Gao D, Driehuis E, Sawyers CL, et al. (2016) Organoid culture systems for prostate epithelial and cancer tissue. Nat Protoc 11: 347-358.

64. DeRose YS, Gligorich KM, Wang G, Georgelas A, Bowman P, et al. (2013) Patient-derived models of human breast cancer: Protocols for in vitro and in vivo applications in tumor biology and translational medicine. Curr Protoc Pharmacol.

65. Garbe JC, Bhattacharya S, Merchant B, Bassett E, Swisshelm K, et al. (2009) Molecular distinctions between stasis and telomere attrition senescence barriers shown by longterm culture of normal human mammary epithelial cells. Cancer Res 69: 7557-7568.

66. Zubeldia Plazaola A, Ametller E, Mancino M, Prats de Puig M, Lopez Plana A, et al. (2015) Comparison of methods for the isolation of human breast epithelial and myoepithelial cells. Front Cell Dev Biol 3: 32.

67. Tilson SG, Haley EM, Triantafillu UL, Dozier DA, Langford CP, et al. (2015) ROCK inhibition facilitates in vitro expansion of glioblastoma stem-like cells. PLoS One 10: e0132823.

68. Welm BE, Dijkgraaf GJP, Bledau AS, Welm AL, Werb Z (2008) Lentiviral transduction of mammary stem cells for analysis of gene function during development and cancer. Cell Stem Cell 2: 90-102.

69. Cheung KJ, Gabrielson E, Werb Z, Ewald AJ (2013) Collective invasion in breast cancer requires a conserved basal epithelial program. Cell 155: 1639-1651.

70. Liu W, Qian N, Li R, Dou K (2010) Replacing Hoechst33342 with rhodamine123 in isolation of cancer stem-like cells from the MHCC97 cell line. Toxicol In Vitro 24: 538-545.

71. Walsh AJ, Cook RS, Sanders ME, Aurisicchio L, Ciliberto G, et al. (2014) Quantitative optical imaging of primary tumor organoid metabolism predicts drug response in breast cancer. Cancer Res 74: 5184-5194.

72. Pradhan S, Hassani I, Seeto WJ, Lipke EA (2017) PEG-fibrinogen hydrogels for three-dimensional breast cancer cell culture. J Biomed Mater Res Part A 105: 236-252.

73. Zhang Z, Christin JR, Wang C, Ge K, Oktay MH, et al. (2016) Mammary-stem-cell-based somatic mouse models reveal breast cancer drivers causing cell fate dysregulation. Cell Rep 16: 3146-3156.

74. Jardé $T$, Lloyd Lewis B, Thomas M, Kendrick H, Melchor L, et al. (2016) Wnt and Neuregulin1/ErbB signalling extends 3D culture of hormone responsive mammary organoids. Nat Commun 7: 13207.

75. Monteiro J, Gaspar C, Richer W, Franken PF, Sacchetti A, et al. (2014) Cancer stemness in Wnt-driven mammary tumorigenesis. Carcinogenesis 35: 2-13. 
76. Wu A, Dong Q, Gao H, Shi Y, Chen Y, et al. (2016) Characterization of mammary epithelial stem/progenitor cells and their changes with aging in common marmosets. Sci Rep 6: 32190 .

77. Zhao M, Sachs PC, Wang X, Dumur Cl, Idowu MO, et al (2012) Mesenchymal stem cells in mammary adipose tissue stimulate progression of breast cancer resembling the basal-type. Cancer Biol Ther 13: 782-792.

78. Sokol ES, Miller DH, Breggia A, Spencer KC, Arendt LM, et al. (2016) Growth of human breast tissues from patient cells in 3D hydrogel scaffolds. Breast Cancer Res 18: 19.

79. Van Lidth de Jeude JF, Vermeulen JLM, Montenegro Miranda PS, Van den Brink GR, Heijmans J (2015) A protocol for lentiviral transduction and downstream analysis of intestinal organoids. J Vis Exp.

80. Fernández Periáñez R, Molina Privado I, Rojo F, Guijarro Muñoz I, Alonso Camino V, et al. (2013) Basement membrane-rich organoids with functional human blood vessels are permissive niches for human breast cancer metastasis. PLoS One 8: e72957.

81. Hicks C, Kumar R, Pannuti A, Miele L (2012) Integrative analysis of response to tamoxifen treatment in ER-positive breast cancer using GWAS information and transcription profiling. Breast Cancer (Auckl) 6: 47-66.

82. Sonnenberg $M$, van der Kuip $H$, Haubeiss $S$, Fritz $P$, Schroth W, et al. (2008) Highly variable response to cytotoxic chemotherapy in carcinoma-associated fibroblasts (CAFs) from lung and breast. BMC Cancer 8: 364.

83. Sowder M, Ludwik K, Pasic L, Brenin D, Stricker T, et al. (2017) Abstract P1-06-05: Breast cancer organoid cultures preserve intra-tumor heterogeneity and reveal intrinsically resistant phenotypes to standard chemotherapies. Cancer Res 77.

84. Velasco Velázquez MA, Homsi N, De La Fuente M, Pestell RG (2012) Breast cancer stem cells. Int J Biochem Cell Bio 44: 573-577.

85. Broutier L, Mastrogiovanni G, Verstegen MM, Francies HE, Gavarró LM, et al. (2017) Human primary liver cancer derived organoid cultures for disease modeling and drug screening. Nat Med 23: 1424-1435.

86. Bruna A, Rueda OM, Greenwood W, Batra AS, Callari M, et al. (2016) A biobank of breast cancer explants with preserved intra-tumor heterogeneity to screen anticancer compounds. Cell 167: 260-274.

87. van de Wetering M, Francies HE, Francis JM, Bounova $\mathrm{G}$, lorio $\mathrm{F}$, et al. (2015) Prospective derivation of a living organoid biobank of colorectal cancer patients. Cell 161 933-945.
88. Torisawa Y, Shiku H, Yasukawa T, Nishizawa M, Matsue T (2005) Multi-channel 3-D cell culture device integrated on a silicon chip for anticancer drug sensitivity test. Biomaterials 26: 2165-2172.

89. Kim C, Bang JH, Kim YE, Lee SH, Kang JY (2012) Onchip anticancer drug test of regular tumor spheroids formed in microwells by a distributive microchannel network. Lab Chip 12: 4135-4142.

90. Kashaninejad N, Nikmaneshi M, Moghadas H, Kiyoumarsi Oskouei A, Rismanian M, et al. (2016) Organ-tumor-on-achip for chemosensitivity assay: A critical review. Micromachines 7: 130 .

91. Kenny PA, Lee GY, Myers CA, Neve RM, Semeiks JR, et al. (2007) The morphologies of breast cancer cell lines in three-dimensional assays correlate with their profiles of gene expression. Mol Oncol 1: 84-96.

92. Calvo F, Ege N, Grande Garcia A, Hooper S, Jenkins RP, et al. (2013) Mechanotransduction and YAP-dependent matrix remodelling is required for the generation and maintenance of cancer-associated fibroblasts. Nat Cell Biol 15: 637-646.

93. Fenner J, Stacer AC, Winterroth F, Johnson TD, Luker KE, et al. (2014) Macroscopic stiffness of breast tumors predicts metastasis. Sci Rep 4: 5512.

94. Eglen RM, Randle DH (2015) Drug discovery goes three-dimensional: Goodbye to flat high-throughput Screening? Assay Drug Dev Technol 13: 262-265.

95. Edmondson R, Broglie JJ, Adcock AF, Yang L (2014) Three-dimensional cell culture systems and their applications in drug discovery and cell-based biosensors. Assay Drug Dev Technol 12: 207-218.

96. Sodek KL, Ringuette MJ, Brown TJ (2009) Compact spheroid formation by ovarian cancer cells is associated with contractile behavior and an invasive phenotype. Int J Cancer 124: 2060-2070.

97. Fatehullah A, Tan SH, Barker N (2016) Organoids as an in vitro model of human development and disease. Nat Cell Biol 18: 246-254.

98. Helgesson G (2012) In defense of broad consent. Cambridge Q Healthc Ethics 21: 40-50.

99. Bunnik EM, Janssens ACJW, Schermer MHN (2013) A tiered-layered-staged model for informed consent in personal genome testing. Eur J Hum Genet 21: 596-601.

100. Boers SN, van Delden JJ, Clevers $H$, Bredenoord AL (2016) Organoid biobanking: Identifying the ethics. EMBO Rep 17: 938-941.

101. Schwank G, Koo BK, Sasselli V, Dekkers JF, Heo I, et al. (2013) Functional repair of CFTR by CRISPR/Cas9 in intestinal stem cell organoids of cystic fibrosis patients. Cell Stem Cell 13: 653-658. 